\title{
CORRECTION
}

\section{Correction to: Role of transabdominal ultrasound in the diagnosis of autoimmune pancreatitis}

\section{Shinji Okaniwa ${ }^{1}$ (D)}

Published online: 5 October 2021

(c) The Japan Society of Ultrasonics in Medicine 2021

\section{Correction to: Journal of Medical Ultrasonics https://doi.org/10.1007/s10396-021-01133-y}

In the original publication of the article, some of the arrows in the Figs. 4, 5, 9, 10, 11, 12 are in red. All the arrows are changed to white in this correction.

The original article can be found online at https://doi.org/10.1007/ s10396-021-01133-y.

Shinji Okaniwa

okaniwa@cocoa.ocn.ne.jp

1 Department of Gastroenterology, Iida Municipal Hospital, 438 Yawata-machi, Iida, Nagano 395-8502, Japan 


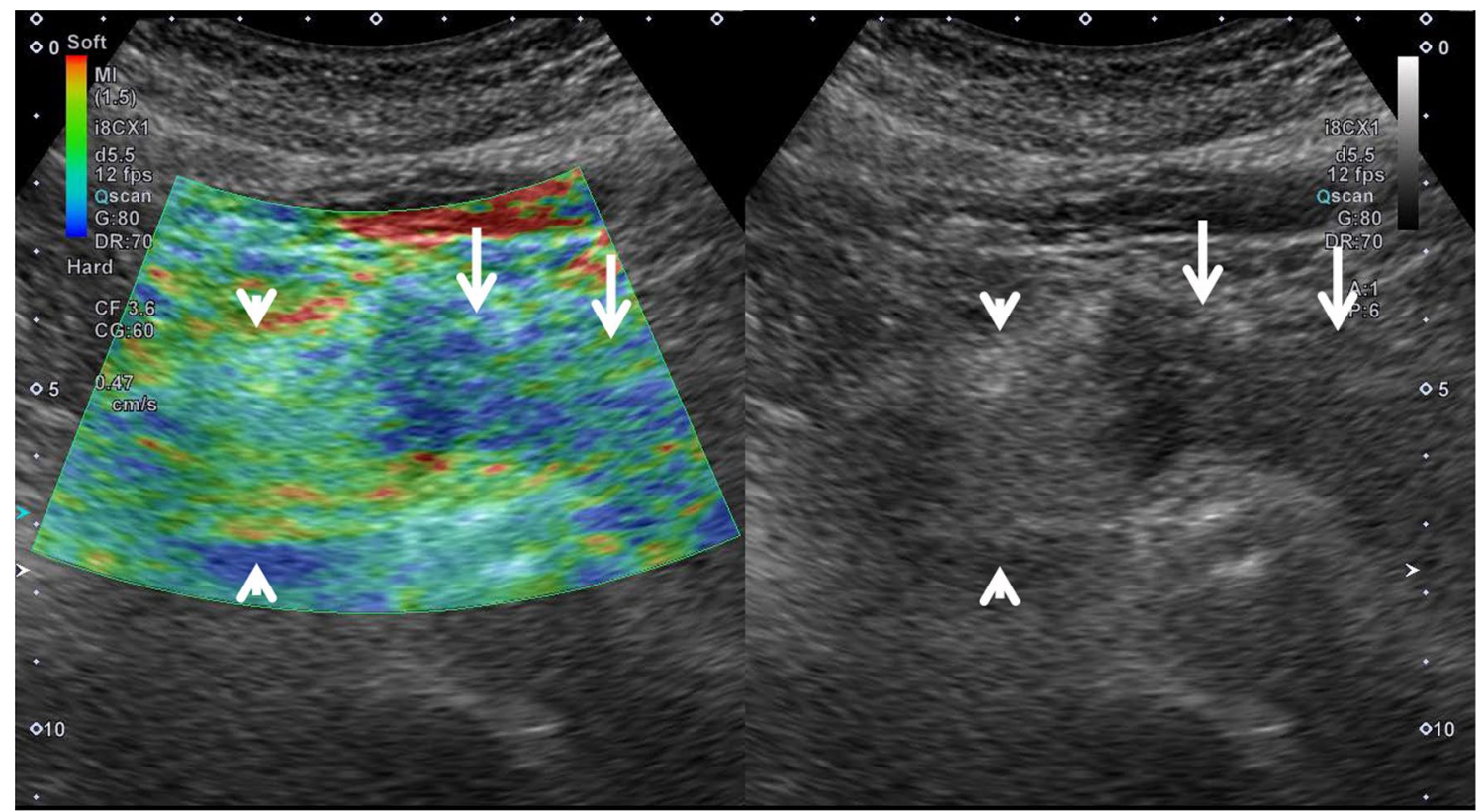

Fig. 4 Images of strain elastography in segmental AIP. Elastography shows a small spotted, mainly blue pattern in the swollen body and tail of the pancreas (arrow) (arrowhead: intact pancreatic head)
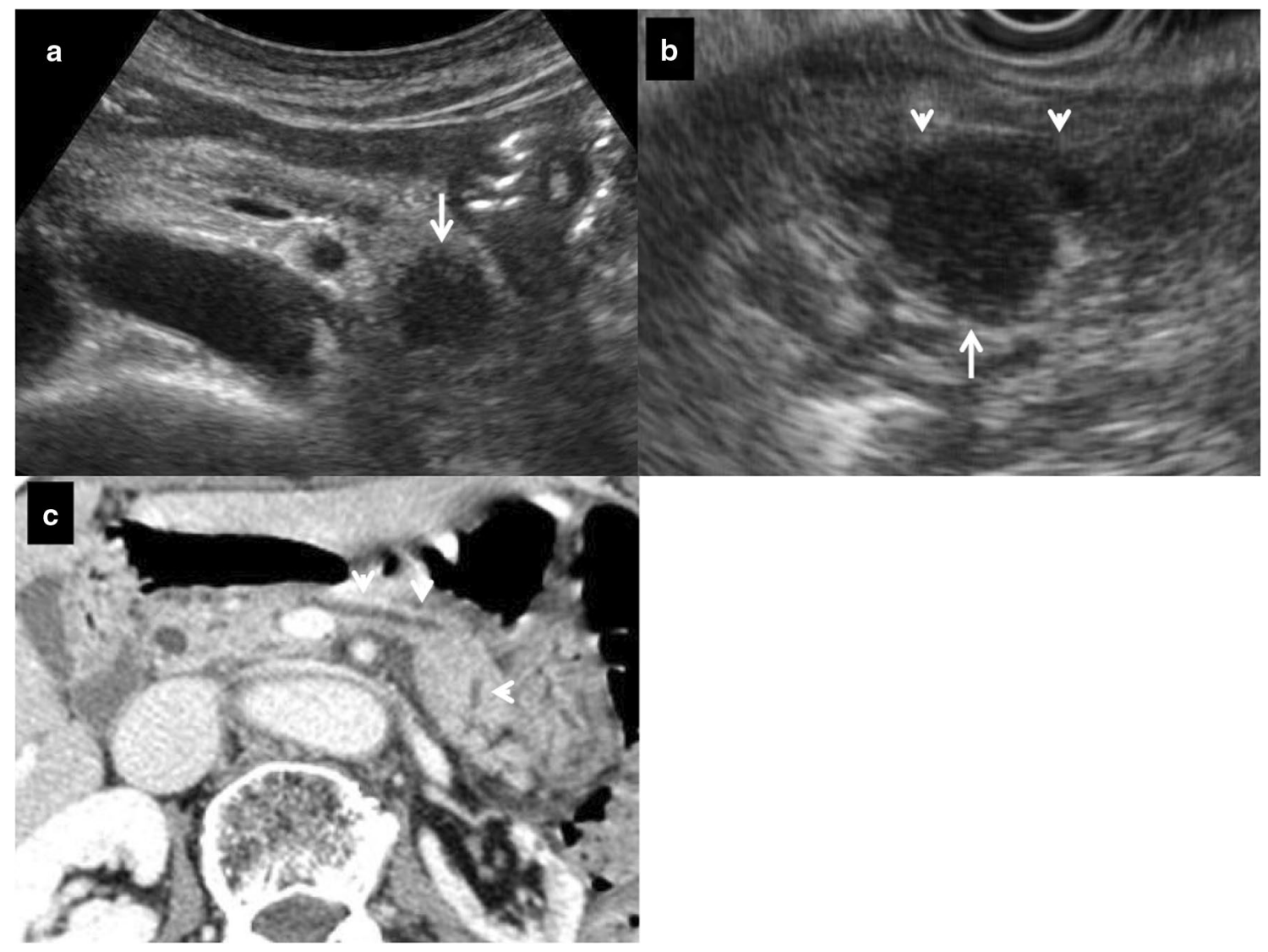

Fig. 5 Images of focal AIP. a US shows a hypoechoic mass lesion in the pancreatic tail (arrow). b EUS shows a hypoechoic solid lesion with an uneven margin (arrow) compressing the main pancreatic duct

(arrowhead). c CT shows only the detoured main pancreatic duct (arrowhead) without an obvious mass lesion 


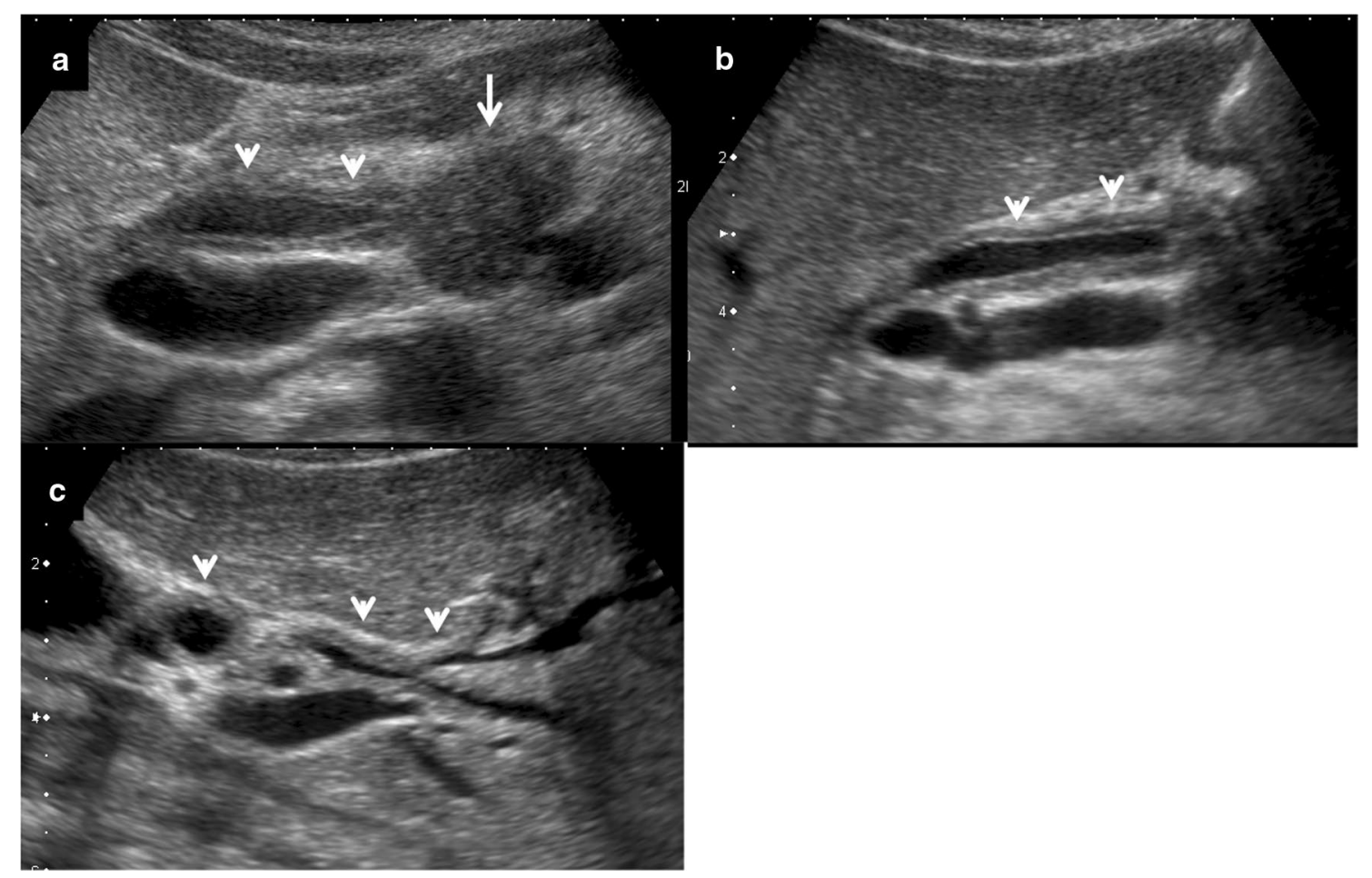

Fig. 9 US images of type2 IgG4-SC. US shows a hypoechoic mass lesion in the pancreatic head (arrow) with a diffusely distributed thickened wall throughout the extrahepatic and intrahepatic bile ducts (arrowheads). a Distal bile duct, b perihilar bile duct, and $\mathbf{c}$ intrahepatic bile duct 



Fig. 10 Images of parenchymal-echo type of IgG4-SC. US shows hypoechoic wall thickening (arrow) mimicking a bile duct carcinoma a and diffusely enlarged hypoechoic pancreatic parenchyma with the penetrating duct sign (arrow) b. ERC shows distal bile duct obstruction (arrow) with multiple pancreatic duct stenosis (arrowhead) (c)



Fig. 11 Bile duct wall thickening without obvious stenosis. The pancreatic head appears severely swollen (arrow), and the dilated extrahepatic bile duct (arrowhead) shows three-layered (high-low-high pattern) wall thickening with a markedly thickened middle layer (a). b Short-axis image using a linear probe (arrowhead: extrahepatic bile duct) 


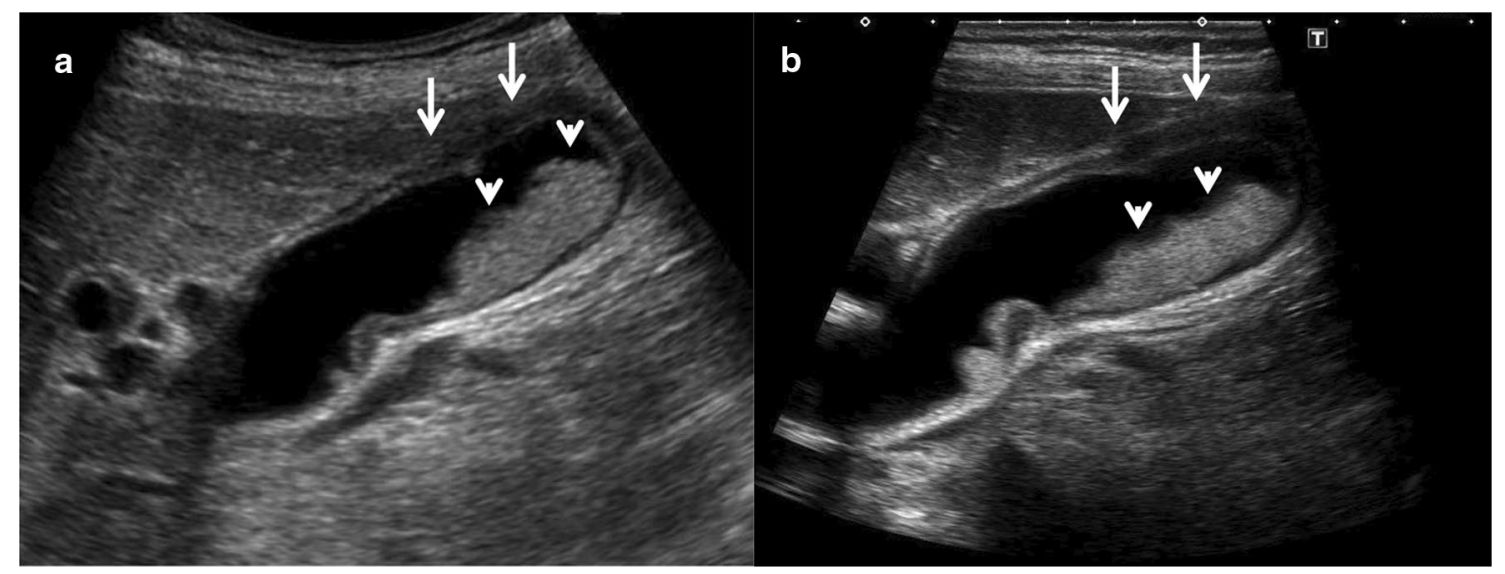

Fig. 12 Images of GB in IgG4-SC. The GB shows marked thickening of the middle layer (arrow) with debris echoes (arrowhead) (a). Image of high-resolution linear probe $(\mathbf{b})$

Publisher's Note Springer Nature remains neutral with regard to jurisdictional claims in published maps and institutional affiliations. 